Die Fragen und Probleme, die im nachstehenden Artikel angesprochen werden, sind grösstenteils angebracht. Obwohl die FMH die meisten dieser Anliegen teilt, unterstützt sie die Idee eines Moratoriums in Bezug auf die Einführung von SwissDRG nicht. Zum einen geht es dabei um die Anwendung eines Gesetzes, das nach den demokratischen Regeln erlassen wurde, und seit dem Beschluss des Parlaments haben sich keine wesentlichen neuen Aspekte ergeben. Zum anderen würde eine Verschiebung des Inkrafttretens der neuen Tarifstruktur nicht zur Lösung der hängigen Probleme beitragen, sondern bloss einige Fristen verlängern. Ganz offensichtlich üben die Wirtschaft und die Versicherer in dieser Sache sehr starken Druck aus und lassen die Realität ausser Acht, mit der die Leistungserbringer und die Patienten konfrontiert sind. Doch statt eine aussichtslose Verweigerungshaltung einzunehmen, muss die Ärzteschaft ständig Präsenz markieren und Einfluss auf die Entscheidungen nehmen, indem sie sich aktiv an der Dynamik beteiligt, die sich innerhalb der SwissDRG AG entwickelt hat. Dass die Ärzteschaft dazu bereit ist, davon zeugt die enorme Arbeit, die unsere Fachgesellschaften in den Antragsrunden im Hinblick auf die Überarbeitung unserer Klassifikation der Interventionen leisten.

Die Einführung der DRG ist ein Katalysator, der bestimmte Rationalisierungsmassnahmen auslösen kann. Diese sind jedoch unab- hängig vom System der Spitalfinanzierung unvermeidlich geworden. Es ist verständlich, dass sich einige von uns von diesen Umstrukturierungsprozessen direkt bedroht fühlen. Verschiedene Kantone arbeiten jedoch schon seit mehreren Jahren mit den DRG. Ihre Erfahrungen zeigen, dass dies für die Patienten wie für die Leistungserbringer nicht mit grösseren Nachteilen verbunden sein muss.

Die Einführung von SwissDRG wird entsprechend dem klaren politischen Willen wie vorgesehen erfolgen. Übrigens hat der Bundesrat am 18. Juni 2010 die Version 0.2 der neuen Tarifstruktur genehmigt. Es ist wichtig, dass wir uns weiterhin für die ständige Optimierung des Systems einsetzen, damit die Version, die bei der Einführung zur Anwendung gelangen wird, so weit als möglich entwickelt ist. Zweifellos werden am 1. Januar 2012 noch nicht alle hängigen Probleme gelöst sein. Um einen reibungslosen Systemwechsel zu ermöglichen, sollte auf nationaler Ebene eine einheitliche Übergangsregelung beschlossen werden.

Dr. med. Pierre-François Cuénoud, Mitglied des Zentralvorstandes der FMH, Verantwortlicher Ressort SwissDRC

\title{
DRG - Aufruf zu einem Moratorium
}

\section{Die Einführung der Swiss-DRGs rückt näher. Das vorgesehene Datum vom 1. Januar 2012 scheint vielen zunehmend unverantwortlich, da manche Punkte unklar bzw. ungelöst sind. Ein Moratorium würde Zeit bringen, die hängigen Fragen zu klären.}

Christian Hess ${ }^{a}$, Urs Strebel ${ }^{b}$

a Dr. med., Chefarzt Medizin Spital Affoltern

b Dr. med., ehemaliger Chefarzt Medizin am Spital Männedorf und Präsident Prüfungskommission SGIM

Korrespondenz:

Dr. med. Christian Hess

Spital Affoltern

Sonnenbergstr. 27

CH-8910 Affoltern am Albis

christian.hess@spitalaffoltern.ch

www.drg-moratorium.ch
Als der Entscheid zur Umstellung auf diagnosebezogene Fallkostenpauschalen gefällt wurde, sprach man vom wichtigen Systemwechsel, weg von der Defizitfinanzierung hin zu klaren Preisen für Leistungen. Man sprach von mehr Transparenz, Vergleichbarkeit unter den Spitälern, mehr Wettbewerb, von verbesserter Qualität, sowie insbesondere von Kosteneinsparungen.

Von diesen Versprechen dürfte wenig übrig bleiben. Die Swiss-DRG AG arbeitet zwar mit enormem Aufwand Kostengewichte auf drei Stellen nach dem Komma aus, die Baserate allerdings liegt immer noch im Dunkeln und wird letztlich weniger ein errechneter Preis als ein politisch ausgehandelter Kompromiss sein. Die Qualität wird, wie in der Schweiz vermutet wird (Berner Studie 2010) und international auch sichtbar ist, sinken, die versprochene kontrollierende Begleitforschung ist nicht aufgegleist. Und schliesslich geht mittlererweile niemand mehr von Kostenersparnissen oder gedämpftem Kostenwachstum aus, ganz im Gegenteil: Kostensteigerungen werden mittlererweile von den meisten zugegeben.

Transparenz und Vergleichbarkeit machen die Patienten nicht gesünder und, dass Wettbewerb zu kei- ner Kostensenkung führt, hat seinen Grund darin, dass das Gesundheitswesen kein üblicher Markt ist. Zudem werden auch im üblichen Markt Preissenkung in aller Regel durch Mengenausweitung erreicht, was für das Gesundheitswesen nicht das Ziel sein kann. Die Tragweite des Projektes Swiss-DRG, das letztlich einem gesundheitspolitischen Bevölkerungsexperiment entspricht, wurde leider von vielen (auch von uns Ärzten) in der Frühphase unterschätzt. Eine öffentliche Diskussion hat nicht stattgefunden, auch nicht, nachdem die Nationale Ethikkommission im Bereich der Humanmedizin NEK in ihrer differenzierten und kritischen Stellungnahme vom September 2008 dringend dazu geraten hat.

Treibende Kräfte sind neben der privat organisierten Swiss-DRG AG (in der beispielsweise der SBK, als grösste Arbeitnehmergruppe im Spitalwesen, mangels finanzieller Mittel nicht vertreten ist) Beratungsfirmen, Gesundheitsökonomen, Kodierfirmen, IT-Unternehmen und Politiker. Es scheint, dass sich ein neuer «Conflict of interest» zwischen Medizin, Beratungsfirmen, IT und anderen Interessensgruppen eingeschlichen hat. Direkt oder indirekt werden alle diese patien- 
tenfernen Dienste von den Leistungserbringern bezahlt, und die Mittel fehlen «an der Front».

Da es aber beim Entscheid für Swiss-DRG um nichts weniger als die Zukunft des Schweizerischen Gesundheitswesens geht, müssen alle ungeklärten Fragen, Sorgen und Probleme einem breiten öffentlichen Diskurs zugeführt werden. In diesem Diskurs geht es auch um die Frage, was für ein Gesundheitssystem sich die
- Schwierig standardisierbare Bereiche wie die Pädiatrie, seltene Krankheiten und die Behandlung von polymorbiden Patientinnen müssen vom DRGSystem ausgenommen und sinnvoll geregelt werden.

- Es muss klar sein, wie die immer wichtiger werdenden psychosozialen Aspekte angemessen berücksichtigt werden können.

\section{In diesem Diskurs geht es auch um die Frage, was für ein Gesundheits- system sich die Schweizer Bevölkerung wünscht, welches Menschenbild ihm hinterlegt sein soll}

Schweizer Bevölkerung wünscht, welches Menschenbild ihm hinterlegt sein soll und was wir alle von ihm erwarten dürfen. Dass bereits 10 Kantone mit Fallkosten abrechnen, ohne dass alle befürchteten negativen Auswirkungen auftreten, ist kein Beweis für das Funktionieren der Swiss-DRG, denn zum einen wird mit AP-DRGs (All Patient Diagnosis related groups = amerikanische Variante) abgerechnet, zum andern sind diese Systeme noch nicht im gleichen Masse «scharf gestellt», wie es bei den Swiss-DRGs vom 1. Januar 2010 vorgesehen ist. Es braucht deshalb ein DRG-Moratorium bis zumindest folgende Fragen und Probleme geklärt sind:

- Die Versorgungssicherheit aller Patientinnen und Patienten muss gewährleistet sein.

- Der Datenschutz darf nicht angetastet werden.

- Die Aus- und Weiterbildung von Ärztinnen und Ärzten sowie des gesamten Gesundheitspersonals muss sichergestellt und finanziert sein.

- Die Arbeitsbedingungen des gesamten Gesundheitspersonals und damit die Qualität der medizinischen und pflegerischen Leistungen dürfen nicht verschlechtert werden. Die verschiedenen Spitaltypen und die regionalen Lohn- und Kostenstrukturen müssen gebührend berücksichtigt werden. In der Pflege ist der Effizienzdruck besonders gross (in Deutschland fielen seit der DRG-Einführung 30000 Vollzeitstellen zum Opfer).

- Die Auswirkungen auf die vor- und nachgelagerten Bereiche (z. B. Hausärztinnen und Hausärzte, Pflegeinstitutionen, Spitex, Rehabilitation- insbesondere Neurorehabilitation) müssen bekannt sein. Es darf keine unkontrollierte Verschiebung der Gesundheitskosten in den ambulanten Bereich mit entsprechender Prämienerhöhung stattfinden.
- Die Kosten, welche die gesamtschweizerische Einführung von DRG verursachen, wie Investitionen in den steigenden Verwaltungsapparat und wiederkehrende Betriebskosten für Kodierung, Kontrollen und Korrekturen müssen transparent geregelt sein und dürfen nicht zu Lasten der direkten Patientenversorgung gehen.

- Die herrschenden vielen Unklarheiten und Unsicherheiten bezüglich Einbezug der Investitionskosten müssen beseitigt und frühzeitig und realistisch geklärt werden.

- Für die definitive Preissetzung (Baserate) müssen die Kriterien klar und transparent festgelegt sein.

- Alle Leistungserbringer - auch Versorger im ambulanten Bereich - müssen in die Umsetzungsarbeiten einbezogen werden.

- Die Begleitforschung muss ein bis zwei Jahre vor Einführung der flächendeckenden Anwendung von DRG in der Schweiz einsetzen.

Mit dem Moratorium wollen wir mehr Zeit haben, um all diese hängigen Fragen hinreichend zu klären, und insbesondere bei diesen versorgungspolitisch relevanten Fragen auch eine notwendige Öffentlichkeitsdiskussion anregen.

Ein überstürzter Systemwechsel wirkt sich vor allem für Patienten und Leistungserbringer am Patientenbett negativ aus.

Wenn unsere Argumente Sie überzeugen, können Sie den Aufruf zum Moratorium unter folgender Internet-Adresse unterschreiben:

www.drg-moratorium.ch 